\title{
Kindlin-2 promotes clear cell renal cell carcinoma progression through the Wnt signaling pathway
}

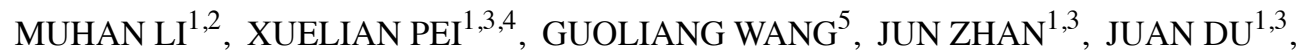 \\ HAO JIANG ${ }^{1,3}$, YAN TANG ${ }^{1,3}$, HONGQUAN ZHANG ${ }^{1,3}$ and HUIYING HE ${ }^{1,2}$ \\ ${ }^{1}$ Key Laboratory of Carcinogenesis and Translational Research, Ministry of Education, \\ and State Key Laboratory of Natural and Biomimetic Drugs, Peking University Health Science Center; \\ ${ }^{2}$ Department of Pathology, Peking University Health Science Center, and Peking University Third Hospital; \\ ${ }^{3}$ Department of Anatomy, Histology and Embryology, Peking University Health Science Center, Beijing 100191; \\ ${ }^{4}$ Department of Histology and Embryology and Key Laboratory of Xinjiang Endemic and Ethnic Diseases, \\ Ministry of Education, Shihezi University School of Medicine, Shihezi, Xinjiang 832000; \\ ${ }^{5}$ Department of Urology, Peking University Third Hospital, Beijing 100083, P.R. China
}

Received January 27, 2017; Accepted June 22, 2017

DOI: 10.3892/or.2017.5789

\begin{abstract}
Kindlin-2 is an integrin-interacting, FERMdomain containing protein, which plays a critical role in tumor progression. However, the specific role of Kindlin-2 in renal cell carcinoma ( $\mathrm{RCC}$ ) progression has not been described. In this study we investigated the role of Kindlin-2 in progression of clear cell RCC (CCRCC), which is the most common RCC subtype, and its underlying mechanisms. Immunohistochemistry studies show that expression of Kindlin-2 in CCRCC is positively correlated with tumor grade, and Kindlin-2 expression in advanced CCRCC with lymph node metastasis was greater than in localized CCRCC. Kindlin-2 expression in CCRCC tumor specimens is also correlated with short patient survival, but is not an independent prognostic factor. Kindlin-2 promotes CCRCC cell migration and invasion in vitro, whereas knockdown of Kindlin-2 inhibited cell migration and invasion. Knockdown of Kindlin-2 also inhibits ACHN cell proliferation in vitro and tumorigenesis in vivo. Kindlin-2 may be required for Wnt pathway activation which underlies the mechanisms of Kindlin-2 promoting CCRCC progression. These findings demonstrate that expression of Kindlin-2 is associated with tumor grade, lymph node metastasis and poor prognosis in CCRCC patients. Kindlin-2 may regulate CCRCC progression through the Wnt signaling
\end{abstract}

Correspondence to: Professor Huiying He, Department of Pathology, Peking University Health Science Center, 38 Xueyuan Road, Haidian, Beijing 100191, P.R. China

E-mail: huiyinghe@bjmu.edu.cn

Professor Hongquan Zhang, Department of Anatomy, Histology and Embryology, Peking University Health Science Center, 38 Xueyuan Road, Haidian, Beijing 100191, P.R. China

E-mail: hongquan.zhang@bjmu.edu.cn

Key words: Kindlin-2, CCRCC, prognosis, migration, invasion, Wnt pathway pathway, promoting CCRCC cell proliferation, migration and invasion.

\section{Introduction}

Renal cell carcinoma (RCC) is the most common kidney cancer, and has become the 7th most common cancer in men in the USA. Over the past two decades, the global incidence of RCC has increased by $2 \%$ per year (1). The incidence and mortality rates of RCC vary widely throughout the world, which may be attributed to environmental and genetic influences (2), but the mortality-to-incidence ratio is highest in Africa and Asia (3). Despite advances in early detection of RCC, 20-30\% of patients still present with metastatic disease at diagnosis, and around one third of patients undergoing nephrectomy for localized tumor eventually develop metastases. RCC is highly heterogeneous in patterns of growth, invasion and metastasis as well as in treatment outcomes (4). Patients with localized RCC tend to have a better prognosis. Despite new targeted therapies, the prognosis for metastatic RCC remains poor, and the 5-year survival rate is $<20 \%(5-7)$. RCC has various histological subtypes. The most common of these is clear cell RCC (CCRCC), which represents $75-80 \%$ of RCC (8), and has a worse outcome than other common types of RCC (4). It is therefore essential to identify molecular markers for translational application in the diagnosis and prognosis of CCRCC.

Kindlin-2 which is encoded by the FERMT2 gene is an integrin-binding focal adhesion protein that is essential in activation of integrin (9). Kindlin-2 not only regulates cell-matrix adhesion and controls cytoskeletal dynamics, but also plays an important role in carcinogenesis (10). High expression of Kindlin-2 can promote tumor progression in prostate cancer, gastric cancer, bladder cancer, lung cancer and malignant mesothelioma (11-15). Kindlin-2 also activates Wnt signaling by forming a tripartite complex with $\beta$-catenin and TCF4 to promote breast cancer cell invasion (16). However, in serous ovarian carcinoma, Kindlin-2 inhibits peritoneal dissemination 
and predicts a more favorable outcome in these patients (17). Kindlin-2 has also been shown to inhibit mesenchymal tumor cell invasion (18). These findings suggest that Kindlin-2 is a molecule that functions in a context-dependent manner. However, little is known about the function of Kindlin-2 in RCC. Here we report that Kindlin-2 expression is significantly correlated with unfavorable prognosis in CCRCC patients and promotes RCC cell migration, invasion and proliferation via activation of Wnt signaling pathway.

\section{Materials and methods}

Patients and samples. Two CCRCC tissue microarrays (TMA) purchased from Shanghai Outdo Biotech (Shanghai, China), which included 109 pairs of tumors and matched peritumoral tissues were used for this study. For each patient, comprehensive pathologic and clinical information was recorded, and anonymity was maintained for all patient information. Tumor grade was determined using the 2016 WHO/ISUP grading system. Pathologic stage was reassigned according to the 2010 American Joint Committee on Cancer TNM Classification. Primary tumors $\left(\mathrm{T}_{\mathrm{x}}\right)$ in four patients and regional lymph nodes $\left(\mathrm{N}_{\mathrm{x}}\right)$ in two patients could not be assessed.

Kindlin-2 immunohistochemistry (IHC). The two TMAs were used for immunostaining analysis of Kindlin-2 protein expression. Deparaffinization and hydration were performed followed by abolition of endogenous peroxidase activity using $3 \%$ hydrogen peroxide for $30 \mathrm{~min}$ and microwave for antigen retrieval in $10 \mathrm{mM}$ sodium citrate buffer ( $\mathrm{pH} \mathrm{6.0)}$ for $20 \mathrm{~min}$. Mouse monoclonal anti-Kindlin- 2 antibody was applied (Millipore, USA) at $2 \mu \mathrm{g} / \mathrm{ml}$ at $4^{\circ} \mathrm{C}$ overnight. The PV9000 2-step plus Poly-HRP Antimouse/rabbit IgG Detection system (Zhongshan Biotech, China) and DAB kit (Zhongshan Biotech) was applied at room temperature. Hematoxylin was used for counterstaining. Non-immune $\operatorname{IgG}$ was used to replace primary antibody as a negative control.

Kindlin-2 immunohistochemical staining was independently assessed by two pathologists blinded to patient outcomes and clinicopathologic parameters. As there was no intrafocal and interfocal heterogeneity in Kindlin-2 protein expression, evaluation of its expression level was based on the intensity of cytoplasmic staining. By using cytoplasmic staining in capillary network of tumor stroma as control, the expressional intensity levels of Kindlin-2 in tumor cells were classified into 3 grades: negative (no brown staining), weak (faint brown staining) and strong (dark brown staining).

Cell culture and establishment of stable cell lines. Human RCC cell lines (ACHN and 769-P) were obtained from the Basic Medical Institute Cell Center, Chinese Academy of Medical Sciences (Beijing, China), and 769-P and ACHN cells were maintained in RPMI-1640 (Hyclone, USA) and minimum essential medium (MEM; Hyclone) respectively, supplemented with $10 \%$ FBS. All cells were cultured in a sterile incubator maintained at $37^{\circ} \mathrm{C}$ with $5 \% \mathrm{CO}_{2}$. Growth media were changed every two days.

Kindlin-2 cDNA was amplified by PCR, inserted into the pLenti6/V5 plasmid, and transfected into 293T cells to obtain a virus.769-P cells were then transduced with the virus. Two days post-transduction, cells were cultured in selection medium with $5 \mu \mathrm{g} / \mathrm{ml}$ blasticidin (Invitrogen, USA) until cells which had not successfully undergone transduction died. Establishment of Kindlin-2 knockdown stable cell lines in ACHN was carried out as described in Zhao et al (19). For transfection, cells were plated in 6 -well plates $\left(1.5 \times 10^{5}\right.$ cells/well $)$ and transfected $24 \mathrm{~h}$ after plating with Lipofectamine 2000 (Roche Applied Science, USA) according to the manufacturer's instructions. Two days post-transfection, cells were cultured in selective medium with addition of $800 \mu \mathrm{g} / \mathrm{ml} \mathrm{G} 418$ (Invitrogen) for selection until cells which were not transfected died.

For signaling pathway inhibitor assay, cells were treated for $24 \mathrm{~h}$ with ILK inhibitor Cpd 22 (Calbiochem, USA) and GSK3 $\beta$ inhibitor LiCl (Sigma, USA).

Cell growth analysis by MTS assay. To assess proliferation, ACHN stable cells $\left(6 \times 10^{3}\right)$ and $769-\mathrm{P}$ stable cells $\left(3 \times 10^{3}\right)$ were seeded into 96 -well plates in $100 \mu \mathrm{l}$ of $10 \% \mathrm{FBS} /$ culture medium and cultured at $37^{\circ} \mathrm{C}$ in a $5 \% \mathrm{CO}_{2}$ incubator. After culturing for $1,2,3,4,5$ or 6 days, $20 \mu 1$ of CellTiter 96 Aqueous One Solution (Promega, USA) was added to each well and followed by incubation for $2 \mathrm{~h}$ at $37^{\circ} \mathrm{C}$ under $5 \% \mathrm{CO}_{2}$. Absorbance was measured at $490 \mathrm{~nm}$ using a microplate reader.

Cell migration and invasion by Transwell assay. Assays were carried out using Transwell chambers $(8 \mu \mathrm{m}$ polycarbonate membrane, Costar, Corning Inc.) that were coated with or without Matrigel (BD Biosciences, USA), and cells were harvested and adjusted to $5 \times 10^{5} / \mathrm{ml}$ using adhesion buffer or culture medium with $0.5 \%$ FBS for separate invasion and migration assays. Cells in $100 \mu 10.5 \%$ FBS culture medium were added to the upper chamber. For migration assays, the lower chamber was coated with $10 \mu \mathrm{g} / \mathrm{ml}$ collagen. After 8-10 h, cells attached to the filter were fixed with methanol and stained with crystal violet $(0.1 \%)$. For the invasion assay, the lower chamber was loaded with culture medium with $20 \%$ FBS. After 24-48 h of incubation, the non-invading cells on the upper side of the chamber were removed. The membranes were fixed with methanol, and stained with Crystal violet. Cells demonstrating migration or invasion were quantified by counting the number of cells in five random fields in each Transwell, and the average cell number was analyzed with the Student's t-test. All experiments were conducted in triplicate and repeated three times.

Real-time PCR. Total cellular RNA was extracted with TRIzol (Invitrogen), and $2 \mu \mathrm{g}$ total RNA were used for reverse transcription with MMLV Reverse Transcriptase (Promega, USA). Real-time PCR was carried out using SYBR Green mix (Applied Biosystems, USA) with PCR conditions as follows: $95^{\circ} \mathrm{C} 3 \mathrm{~min} ; 95^{\circ} \mathrm{C} 20 \mathrm{sec}, 60^{\circ} \mathrm{C} 1 \mathrm{~min}$, for 40 cycles. Gene expression was determined by the comparative CT method $\left(2^{-\Delta \Delta c t}\right)$. All genes were normalized to actin levels. Quantitative PCR was analyzed in triplicate and all experiments were repeated three times.

Tumor formation in vivo. ACHN control cells and Kindlin-2 depleted cells $\left(2 \times 10^{6}\right)$ were counted and resuspended in $100 \mu 1$ MEM medium, then injected subcutaneously into 4-week-old male nude mice (Center of Experimental Animals, Peking 
University, Beijing, China), which were sacrificed 11 weeks after implantation. Tissues from subcutaneous xenografts were used for histologic and immunostaining examination. Mice were maintained according to the Peking University Guidelines of Animal Experiments.

Western blot analyses and reagents. Cells were washed with ice-cold PBS and lysed in a PBS-TDS buffer [PBS with $1 \%$ Triton X-100, $0.5 \%$ sodium deoxycholate, $0.1 \%$ sodium dodecyl sulfate (SDS) $1 \mathrm{mM}$ EDTA, $1 \mathrm{mM}$ phenylmethanesulfonyl fluoride (PMSF), 1 complete inhibitor cocktail (Boehringer)], and centrifuged at $15,000 \mathrm{~g}$ for $20 \mathrm{~min}$ at $4^{\circ} \mathrm{C}$ to obtain a clear lysate. Samples were heated at $95^{\circ} \mathrm{C}$ for $5 \mathrm{~min}$ and then separated on SDS-PAGE gels and blotted onto PVDF membranes. Primary antibodies against Kindlin-2 (Millipore), active $\beta$-catenin (Millipore), $\beta$-catenin (Santa Cruz Biotechnology, USA), TCF4 (Millipore), Snail (Cell Signaling Technology, USA), $\beta$-actin (Zhongshan Biotech) were incubated with these membranes separately under rotation. After thorough washing, membranes were further incubated with corresponding secondary antibodies recognizing either rabbit or mouse Ig (Jackson ImmunoResearch, USA). Bands were visualized with enhanced chemiluminescence (Pierce, USA).

Affymetrix GeneChip human Gene 1.0 ST array. cDNA from ACHN control cells and Kindlin-2 depleted cells were hybridized to Affymetrix GeneChip Human Gene 1.0 ST arrays (Affymetrix, USA). These arrays analyze the expression level for 28,869 transcripts and variants, including 28,132 well-identified human genes by Ensemble. Data were analyzed with GeneChip Operating Software 1.4. GO and pathway analysis derived from KEGG was carried out.

Statistical analyses. The Student's t-test was used for statistical analysis for paired samples. Statistical analysis for patient materials was performed with the Chi-square test, the Kruskal-Wallis test and the Pearson's correlation coefficient. Patient survival was calculated using Kaplan-Meier analysis and comparisons were made using the log-rank test. Univariate and multivariate survival data were analyzed using Cox's proportional hazard model. Variables associated with overall survival at a significance level of $\mathrm{P}<0.1$ on univariate analysis were included in multivariate modelling using backward conditional regression. Overall survival was defined as time from the date of surgery to the date of death from any cause. All statistical analysis employed SAS version 9.1. Results were considered statistically significant at $\mathrm{P}<0.05$.

\section{Results}

Expression of Kindlin-2 correlates with higher tumor grade, lymph node metastasis and poor prognosis in CCRCC patients. To evaluate the involvement of Kindlin-2 in CCRCC progression, IHC analysis of Kindlin-2 was performed on a cohort which included 109 CCRCC patients. For a control, we found Kindlin-2 is highly expressed in the glomerulus and distal convoluted renal tubules, while its expression is generally reduced in proximal convoluted tubules as shown in Fig. 1A-a. In CCRCC, Kindlin-2 was highly expressed in tumor stroma, especially in the capillary network. Kindlin-2 expression rate

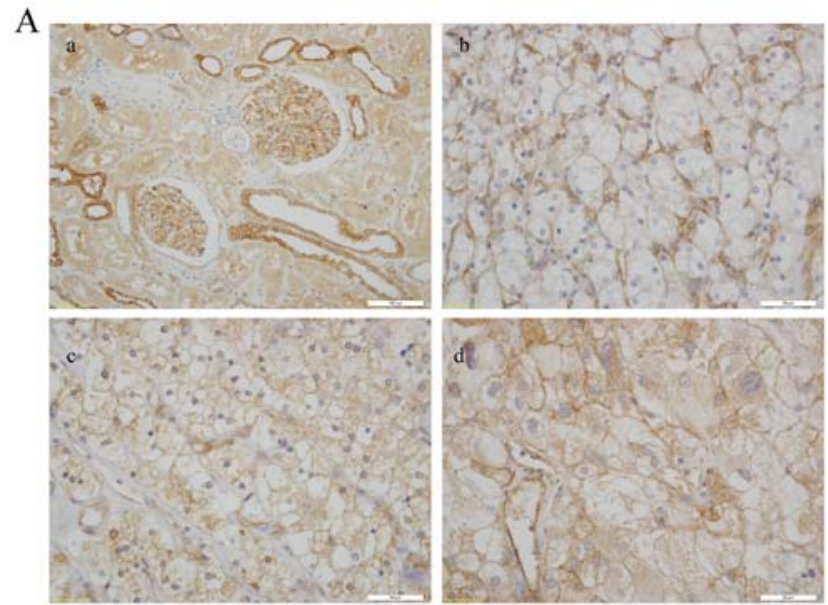

B

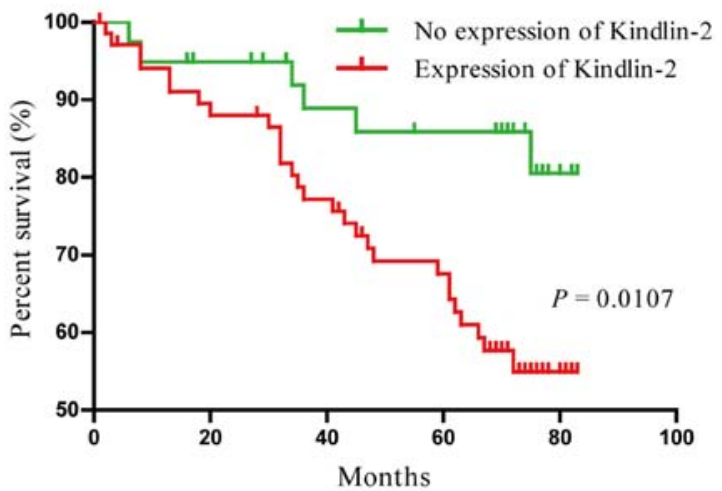

Figure 1. Kindlin-2 expression in CCRCC and Kaplan-Meier survival analysis. (A) Immunohistochemistry evaluation of Kindlin-2 expression in RCC specimens. a, Paraneoplastic kidney tissue (bar, $100 \mu \mathrm{m}$ ) showed strong Kindlin-2 expression, especially in the glomeruli and distal convoluted renal tubules. b-d, Kindlin-2 expression in CCRCC (bar, $50 \mu \mathrm{m}$ ). b, Negative. c, Weak. d, Strong immunoreactivity] (B) Compared to cases without Kindlin-2 expression, CCRCC patients with Kindlin-2 expression showed shorter overall survival $(\mathrm{P}=0.0107)$.

$(84.38 \%, 27 / 32)$ and intensity [weak staining in $40.63 \%(13 / 32)$ and strong staining in $43.75 \%$ (14/32)] were much higher in high-grade tumors (Fuhrman grade 3 and 4 ) than in low-grade tumors (Fuhrman grade 1 and 2). Low grade tumors showed an expression rate of $55.84 \%$ (43/77), and rates for weak staining and strong staining were $38.96 \%$ (30/77) and $16.88 \%$ (13/77) respectively ( $\mathrm{P}=0.0101$, Table I, Fig. 1A-b-d). In addition, advanced tumors with regional lymph node metastasis showed stronger staining for Kindlin-2 than localized cases of CCRCC $(\mathrm{P}=0.0227$, Table I). These results indicate the involvement of Kindlin-2 in the process of CCRCC progression.

To determine whether Kindlin-2 expression is correlated with patient prognosis, survival analysis was used to evaluate patients with available follow-up data of up to 82 months. Among 109 patients, 34 (31.19\%) patients died during follow-up, $66(60.55 \%)$ patients were alive, and $9(8.26 \%)$ patients were lost to follow-up. The median survival time was 69 months. Kaplan-Meier plots and log-rank tests showed that CCRCC patients with Kindlin-2 expression had shorter overall survival than patients without Kindlin- 2 expression $(\mathrm{P}=0.0107$, Fig. 1B). There was no apparent difference in overall survival among patients with different intensities of Kindlin-2 staining $(\mathrm{P}=0.5412$, data not shown). Upon univariate analysis (Table II), Kindlin-2 (HR, 0.41; 95\% CI, 0.20-0.81; P=0.0107), 
Table I. Relationship between Kindlin-2 expression and various clinicopathological features in CCRCC patients.

\begin{tabular}{|c|c|c|c|c|c|}
\hline \multirow[b]{2}{*}{ Clinicopathological features } & \multirow[b]{2}{*}{$\mathrm{N}=109$} & \multicolumn{3}{|c|}{ Kindlin-2 expression } & \multirow[b]{2}{*}{ P-value } \\
\hline & & Negative (\%) & Weak $(\%)$ & Strong (\%) & \\
\hline \multicolumn{6}{|l|}{ Sex } \\
\hline Male & 67 & $21(31.34)$ & $30(44.78)$ & $16(23.88)$ & \multirow[t]{2}{*}{0.502} \\
\hline Female & 42 & $18(42.86)$ & $13(30.95)$ & $11(26.19)$ & \\
\hline \multicolumn{6}{|l|}{ Age, years } \\
\hline$\leq 60$ & 62 & $23(37.10)$ & $22(35.48)$ & $17(27.42)$ & \multirow[t]{2}{*}{0.875} \\
\hline$>60$ & 47 & $16(34.04)$ & $21(44.68)$ & $10(21.28)$ & \\
\hline \multicolumn{6}{|l|}{ AJCC stage } \\
\hline $\mathrm{X}$ & 6 & $2(33.33)$ & $3(50.00)$ & $1(16.67)$ & \multirow{4}{*}{0.0538} \\
\hline I & 64 & $26(40.63)$ & $26(40.63)$ & $12(18.75)$ & \\
\hline II & 20 & $6(30.00)$ & $8(40.00)$ & $6(30.00)$ & \\
\hline III-IV & 19 & $5(26.32)$ & $6(31.58)$ & $8(42.10)$ & \\
\hline \multicolumn{6}{|l|}{ Nuclear grade (Fuhrman) } \\
\hline 1 & 36 & $15(41.67)$ & $14(38.89)$ & $7(19.44)$ & \multirow[t]{3}{*}{$0.0101^{*}$} \\
\hline 2 & 41 & $19(46.34)$ & $16(39.02)$ & $6(14.63)$ & \\
\hline 3 and 4 & 32 & $5(15.63)$ & $13(40.63)$ & $14(43.75)$ & \\
\hline \multicolumn{6}{|l|}{ pT stage } \\
\hline $\mathrm{T}_{\mathrm{x}}$ & 4 & $1(25.00)$ & $3(75.00)$ & $0(0.00)$ & \multirow{4}{*}{0.0596} \\
\hline $\mathrm{T}_{1}$ & 68 & $28(41.18)$ & $26(38.24)$ & $14(20.59)$ & \\
\hline $\mathrm{T}_{2}$ & 20 & $6(30.00)$ & $8(40.00)$ & $6(30.00)$ & \\
\hline $\mathrm{T}_{3}$ and $\mathrm{T}_{4}$ & 17 & $4(23.53)$ & $6(35.29)$ & $7(41.18)$ & \\
\hline \multicolumn{6}{|l|}{$\mathrm{N}$ stage } \\
\hline $\mathrm{N}_{\mathrm{x}}$ & 2 & $1(50.00)$ & $0 \quad(0.00)$ & $1(50.00)$ & \multirow{3}{*}{$0.0227^{*}$} \\
\hline $\mathrm{N}_{0}$ & 99 & $37(37.37)$ & $41(41.41)$ & $21(21.21)$ & \\
\hline $\mathrm{N}_{1}$ & 8 & $1(12.50)$ & $2(25.00)$ & $5(62.50)$ & \\
\hline
\end{tabular}

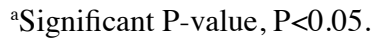

Table II. The effect of clinicopathological characteristics on overall survival by Cox regression analyses.

\begin{tabular}{|c|c|c|c|c|}
\hline \multirow[b]{3}{*}{ Variable } & \multicolumn{4}{|c|}{ OS } \\
\hline & \multicolumn{2}{|c|}{ Univariate analysis } & \multicolumn{2}{|c|}{ Multivariate analysis } \\
\hline & $\operatorname{HR}(95 \% \mathrm{CI})$ & P-value & $\mathrm{HR}(95 \% \mathrm{CI})$ & P-value \\
\hline Kindlin-2 (negative vs. positive) & $0.41(0.20-0.81)$ & 0.0107 & $0.59 \quad(0.23-1.52)$ & 0.276 \\
\hline Age years $(<60$ vs. $\geq 60)$ & $0.53(0.27-1.05)$ & 0.0703 & $0.51 \quad(0.23-1.11)$ & 0.091 \\
\hline Sex (male vs. female) & $1.03(0.52-2.03)$ & 0.943 & - & - \\
\hline Fuhrman grade (1-2 vs. $3-4)$ & $0.04(0.02-0.10)$ & $<0.0001$ & $0.14 \quad(0.06-0.30)$ & $0.000^{\mathrm{a}}$ \\
\hline pT stage (1 2 vs. 3 4) & $0.07(0.02-0.28)$ & 0.0002 & $4.17(0.53-32.68)$ & 0.174 \\
\hline $\mathrm{N}$ stage (0 vs. 1$)$ & $0.000029(0.000001-0.0008)$ & $<0.0001$ & $0.68 \quad(0.11-4.12)$ & 0.678 \\
\hline AJCC stage (1-2 vs. $3-4)$ & $0.03(0.01-0.13)$ & $<0.0001$ & $0.07 \quad(0.01-0.74)$ & $0.028^{\mathrm{a}}$ \\
\hline
\end{tabular}



age (HR, 0.53; 95\% CI, 0.27-1.05; $\mathrm{P}=0.0703)$, Fuhrman grade (HR, 0.04; 95\% CI, 0.02-0.10; P<0.0001), pT stage (HR, 0.07; 95\% CI, 0.02-0.28; $\mathrm{P}=0.0002), \mathrm{N}$ stage (HR, 0.000029; 95\% CI, 0.000001-0.0008; $\mathrm{P}<0.0001)$, and AJCC stage
(HR, 0.03; 95\% CI, 0.01-0.13; P<0.0001) were associated with overall survival and these factors were included in multivariate analysis. Multivariate analysis (Table II) showed that Fuhrman grade (HR, 0.14; 95\% CI, 0.06-0.30; P<0.0001) and AJCC 
A

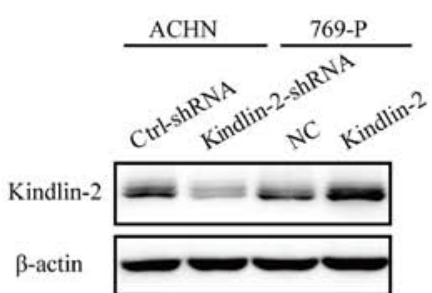

B
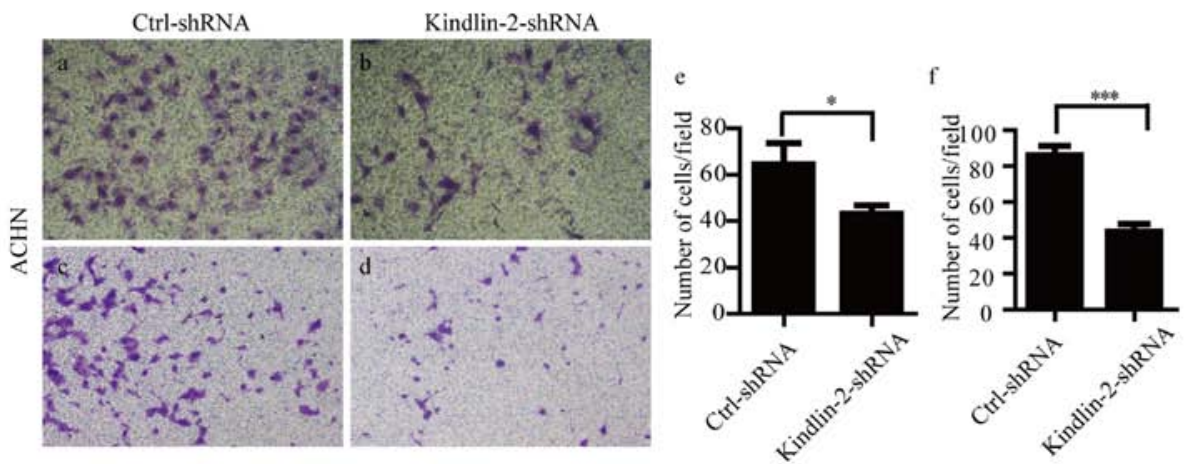

$\mathrm{C}$
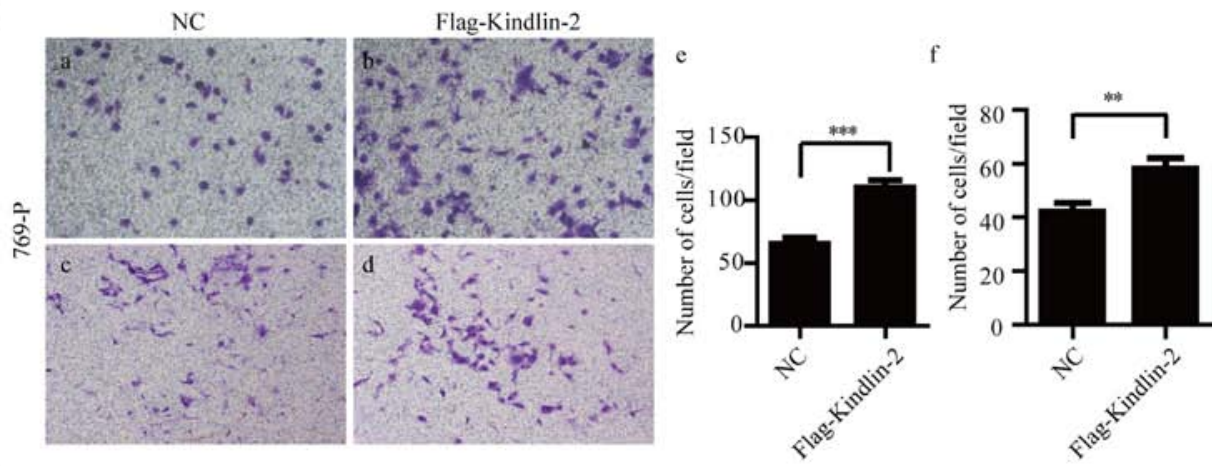

D

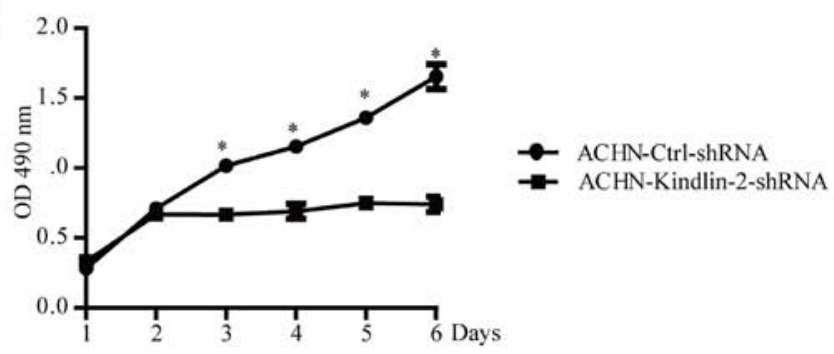

Figure 2. Kindlin-2 expression in stable transfected cell lines and its effects on migration, invasion and proliferation in RCC cell lines. (A) Kindlin-2 expression in knockdown model ACHN cells and in overexpressing model 769-P cells. Transwell migration and invasion assays were used to evaluate the migration and invasion capacity of RCC cells with Kindlin-2 stable overexpression or knockdown. (B) Migration and invasion capacity of stable Kindlin-2 knockdown cells (ACHN-Ctrl-shRNA and ACHN-Kindlin-2-shRNA cells). (C) Migration and invasion capacity of stable Kindlin-2 overexpressing cells (769-P-NC and 769-P-Kindlin-2). (D) MTS proliferation assay showed knockdown of Kindlin-2 reduces cell proliferation in ACHN cells. ${ }^{*} \mathrm{P}<0.05,{ }^{* *} \mathrm{P}<0.01,{ }^{* * *} \mathrm{P}<0.001$.

stage (HR, 0.07; 95\% CI, 0.01-0.74; $\mathrm{P}=0.028$ ) were independently associated with overall survival. However, Kindlin-2 had a P-value of 0.276 and was therefore not an independent prognostic factor in CCRCC. These results indicate that the presence of Kindlin-2 is a factor that predicts poor overall survival in CCRCC patients.

Kindlin-2 promotes cell migration, invasion and proliferation in CCRCC in vitro. Kindlin-2 expression showed significant correlation with tumor grade and patient prognosis, which raised the possibility it plays a role in CCRCC progression. To evaluate this possibility, we chose two CCRCC cell lines, 769-P and ACHN for further study. 769-P cells stably overexpressing Kindlin-2 following retroviral transduction, and ACHN cells with stable knockdown of Kindlin-2 were established (Fig. 2A).
Stable depletion of Kindlin-2 by shRNA inhibited the migratory $(\mathrm{P}=0.0352)$ and invasive $(\mathrm{P}<0.0001)$ capacity of $\mathrm{ACHN}$ cells (Fig. 2B). In comparison, overexpression of Kindlin-2 in 769-P cells significantly promoted potential for cell migration $(\mathrm{P}<0.0001)$ and invasion $(\mathrm{P}=0.0043)$ (Fig. 2C). Depletion of Kindlin-2 in ACHN cells reduced cell proliferation compared to the control group $(\mathrm{P}=0.0009$ on the sixth day as shown in Fig. 2D. However, there was no significant difference in 769-P cell growth in the Kindlin-2 overexpression group versus the control group (data not shown).

Knockdown of Kindlin-2 inhibits CCRCC cell proliferation in vivo. Observation of Kindlin-2 in RCC specimens and RCC cell lines in vitro raised a question as to whether Kindlin-2 plays a role in the regulation of tumor growth in vivo. As 
A

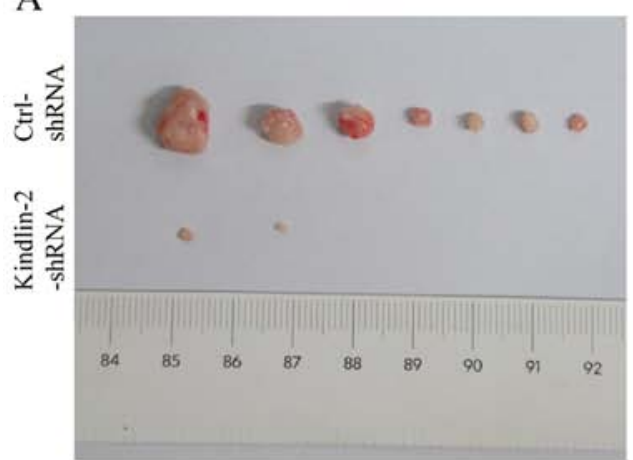

B

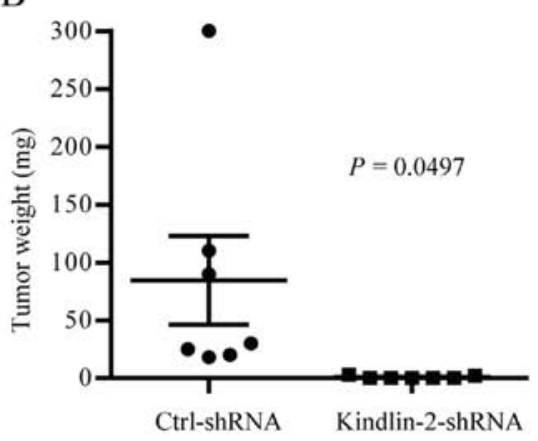

$\mathrm{C}$

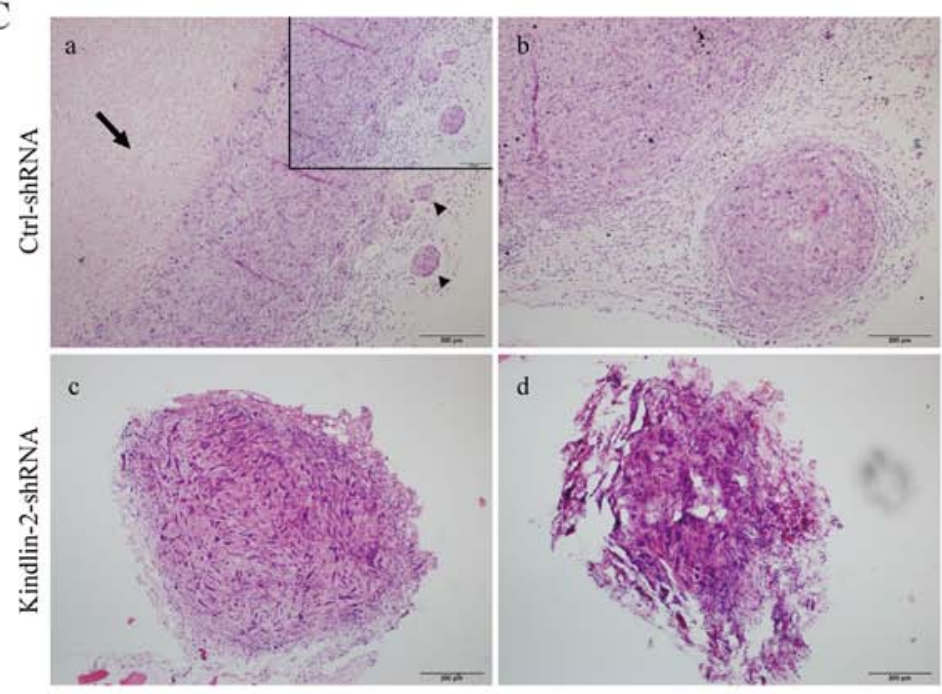

$\mathrm{D}$

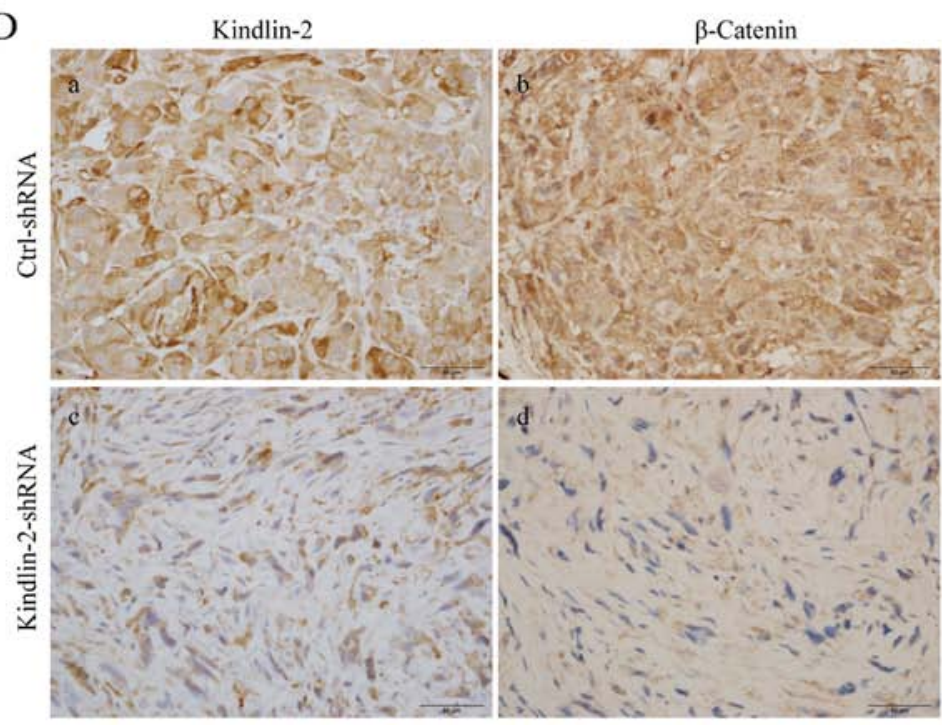

Figure 3. Tumor growth in implanted xenograft was inhibited by Kindlin-2 knockdown. (A) Tumors recovered from mice at day 80. (B) Tumor weight in the ACHN-Kindlin-2-shRNA group was significantly less than in the ACHN-ctrl-shRNA group ( $\mathrm{P}<0.05$ as analyzed by the Student's $t$-test). (C) H\&E (bar, $200 \mu \mathrm{m}$ ) staining in tumors of two groups [a and b, in the ACHN-ctrl-shRNA group, central necrosis (arrow) was frequently observed and there were intravenous tumor emboli (arrowheads) in the no. 4 nude mouse, and a satellite tumor nodule (b) in the no. 1 nude mouse. $c$ and d, Xenograft tumors in no. 2 and no. 7 nude mice of the ACHN-Kindlin-2-shRNA group]. (D) Kindlin-2 and $\beta$-catenin immunostaining in tumors of two groups (Kindlin-2 and $\beta$-catenin in tumors of the ACHN-Kindlin-2-shRNA group showed much lower expression than that of the ACHN-Ctrl-shRNA group).

expected, mouse tumor xenografts with Kindlin-2 knockdown grew significantly more slowly, and tumor size was significantly smaller than that of the control group (Fig. 3A and B). In addition, massive central tumor necrosis, intravenous tumor emboli and peritumoral satellite nodules were found in the control group, but these phenomena which reflect tumor aggressiveness were absent in the ACHN-Kindlin-2-shRNA group (Fig. 3C). Lung and liver metastasis were not observed in either group. We found that $\beta$-catenin immunohistochemical staining was markedly reduced in Kindlin-2 depleted tumor xenografts compared with counterpart controls (Fig. 3D), which stimulated consideration of the Kindlin-2 downstream. 
A

Dif gene signaling pathway (-lgP)
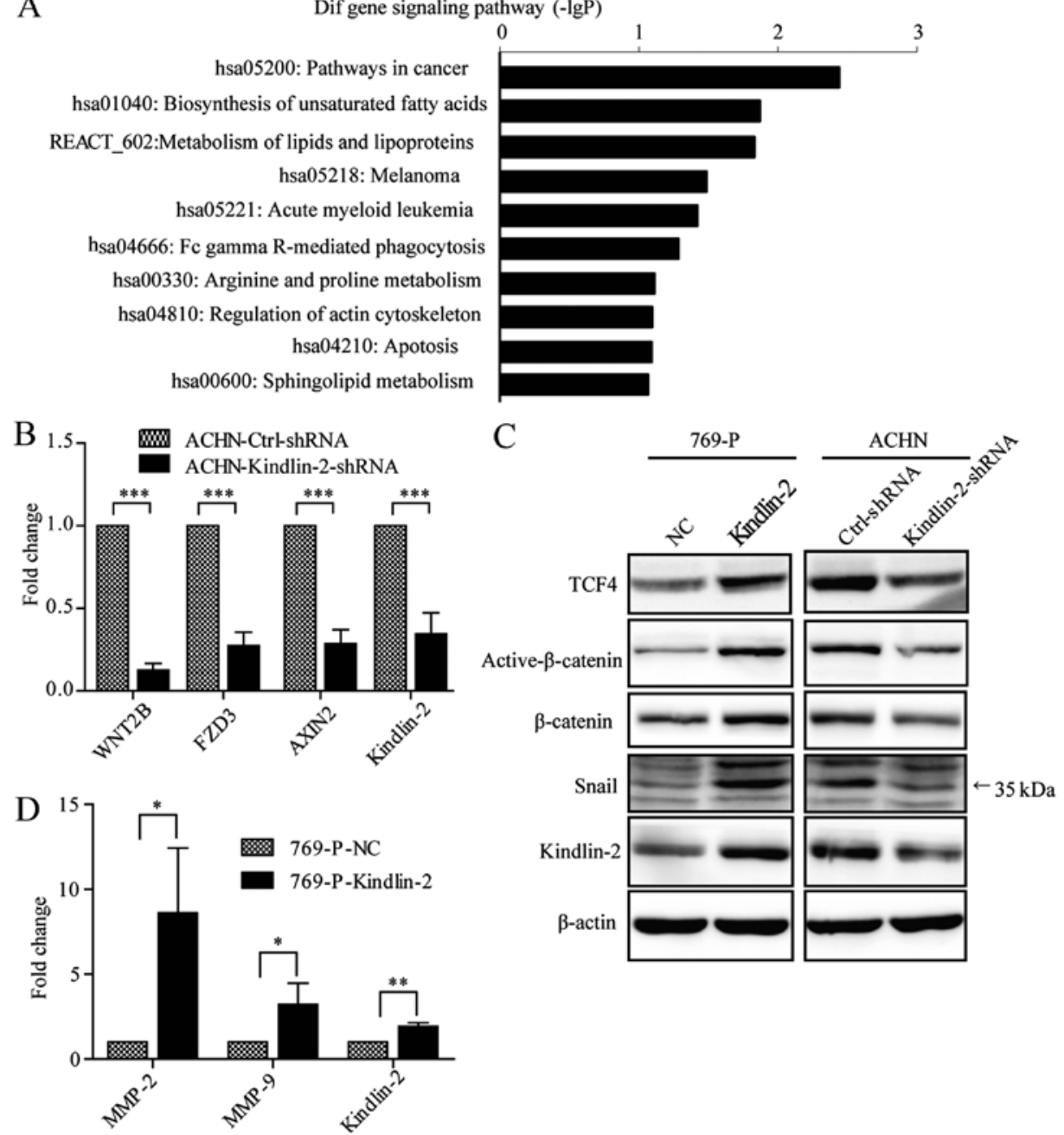

hsa01040: Biosynthesis of unsaturated fatty acids REACT_602:Metabolism of lipids and lipoproteins hsa05218: Melanoma

hsa05221: Acute myeloid leukemia

$\mathrm{h}_{\mathrm{Sa}}$ 04666: Fc gamma R-mediated phagocytosi hsa00330: Arginine and proline metabolism hsa04810: Regulation of actin cytoskeleton hsa04210: Apotosis hsa00600: Sphingolipid metabolism
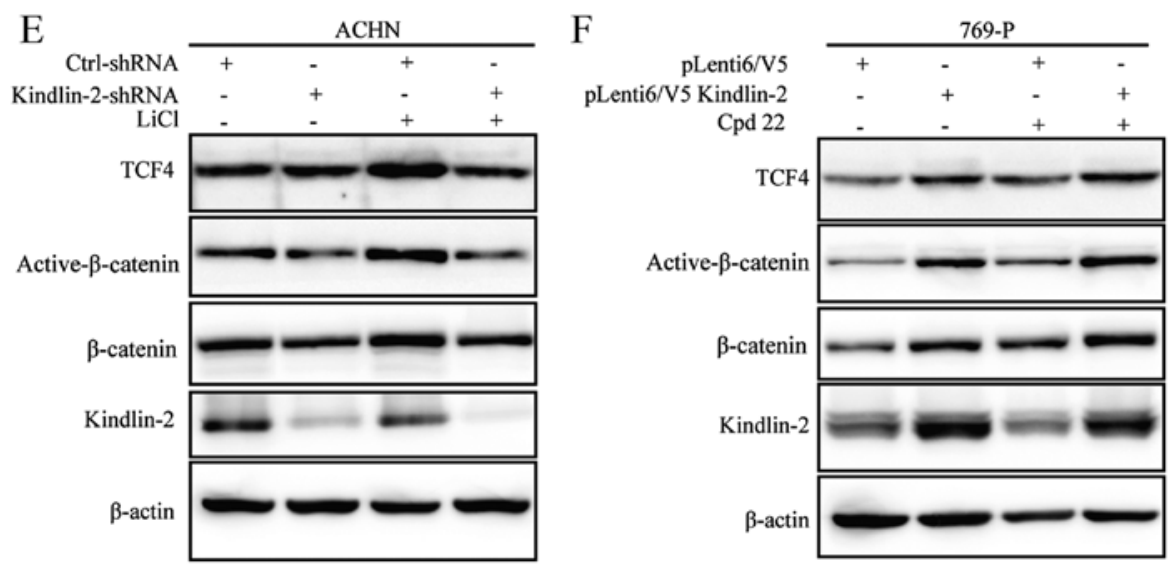

Figure 4. Kindlin-2 promoted CCRCC progression by activating the Wnt signaling pathway. (A) Significantly downregulated pathways targeted by Kindlin-2 knockdown. The y-axis showed pathways with significant alteration and the x-axis, - $\mathrm{LgP}$ (negative logarithm of P-value). Larger -LgP indicated a smaller $\mathrm{P}$-value (based on the KEGG database, pathways with $\mathrm{P}<0.05$ and FDR $<0.05$ were identified as significantly altered pathways). (B) Relative mRNA expression of genes selected in Pathways in cancer of KEGG database was validated by real-time PCR in Kindlin-2 depleted ACHN cells. (C) Western blot analysis of TCF4, $\beta$-catenin, active $\beta$-catenin and Snail expression in Kindlin-2 overexpressing 769-P cells and ACHN knockdown cells. $\beta$-actin was used as a loading control. (D) Relative mRNA expression of MMP-2 and MMP-9 was evaluated by real-time PCR in Kindlin-2 overexpressing 769-P cells. (E and F) Levels of TCF4, $\beta$-catenin and active $\beta$-catenin were evaluated by western blot analysis in (E) Kindlin-2 knockdown ACHN cells treated with LiCl for $24 \mathrm{~h}$ and (F) Kindlin-2 overexpressing 769-P cells with ILK inhibitor Cpd22 treatment for $24 \mathrm{~h}$.

Kindlin-2 activates the Wnt signaling pathway to promote CCRCC progression. To evaluate the mechanism by which Kindlin-2 promotes CCRCC progression, ACHN control cells and Kindlin-2 depleted cells were analyzed with the Affymetrix GeneChip human Gene 1.0 ST array. The detailed
cDNA expression data can be accessed on GEO GSE76020. Based on the KEGG database, pathways in cancer was the most significantly downregulated pathway targeted by Kindlin-2 depletion (Fig. 4A). Among numerous tumorassociated genes, we found that expression of genes involved 
in the Wnt signaling pathway were altered significantly. To confirm these GeneChip results, the alteration of expression of selected genes in the Wnt pathway (WNT2B, FZD3 and Axin2) were validated with real-time PCR (Fig. 4B). Kindlin-2 combines with $\beta$-catenin and TCF4 to activate the Wnt pathway (16), and expression of TCF4, $\beta$-catenin, active $\beta$-catenin and Snail was found in both Kindlin-2 overexpressing 769-P cells and Kindlin-2 depleted ACHN cells. Overexpression of Kindlin-2 elevated the expression of these four genes, whereas knockdown of Kindlin-2 inhibited their expression as shown in Fig. 4C. MMP-2 and MMP-9 are well known target genes of Wnt pathway, and overexpression of Kindlin-2 increased the mRNA level of MMP-2 and MMP-9 in 769-P cells (Fig. 4D).

To further determine whether Kindlin-2 is involved in Wnt pathway activation, ACHN stable cells were treated for $24 \mathrm{~h}$ with $\mathrm{LiCl}$ which is a specific GSK3 $\beta$ inhibitor. $\mathrm{LiCl}$ elevated expression of TCF4, $\beta$-catenin and active $\beta$-catenin in ACHN-Ctrl-shRNA cells (Fig. 4E, compare lanes 1 with 3), showing that $\mathrm{LiCl}$ activates the Wnt pathway in ACHN cells. However, treatment of ACHN stable Kindlin-2 depleted cells with $\mathrm{LiCl}$ did not result in a change of expression of TCF4, $\beta$-catenin or active $\beta$-catenin (Fig. 4E, compare lanes 2 with 4). These findings argue that knockdown of Kindlin-2 inhibits Wnt pathway activation, and raises the possibility Kindlin-2 is required for Wnt pathway activation. Kindlin-2 is known to bind integrin-linked kinase (ILK) for regulation of integrinmediated cell adhesion $(20,21)$, which raises a question as to whether Kindlin-2 regulation of Wnt signaling is mediated by ILK. To address this question, 769-P stable Kindlin-2 overexpressing cells were treated with the ILK inhibitor Cpd 22 for $24 \mathrm{~h}$, however, expression of TCF $4, \beta$-catenin and active $\beta$-catenin did not change significantly in 769-P stable cells treated with Cpd 22 (Fig. 4F). This finding indicates that Kindlin-2 is not dependent on ILK binding for regulation of the Wnt signaling pathway.

\section{Discussion}

Kindlin-2 is being known for the multiple roles it plays in various cancers (11-18). However, to date there has been no detailed study of Kindlin-2 function in RCC. In this study, we evaluated Kindlin-2 expression and focused on its role in CCRCC. CCRCC is the major subtype of RCC, and accounts for approximately $80 \%$ of renal carcinomas. The prognosis for this subtype is poorer than the other two common RCC subtypes including papillary RCC and chromophobe RCC (8).

Higher Kindlin-2 expression in CCRCC is associated with higher nuclear grades and local lymph node metastasis. Although CCRCC patients with Kindlin-2 tumor expression have poor survival, Kindlin-2 expression is not an independent prognostic factor. Kindlin-2 showed higher expression in CCRCC paraneoplastic kidney tissues than that in the tumor itself, and this paradoxical phenomenon raises the possibility Kindlin-2 is involved in CCRCC progression, but not in tumor formation. Recently Yan et al found that increased Kindlin-2 expression was associated with advanced stage, hematogenous metastasis and short survival in CCRCC patients (22). To our surprise, all tumor cases showed Kindlin-2 expression of different intensity in their study, with absent expression in adjacent renal contex tissues. It is oppsite to our finding and earlier observation of Kindlin-2 in adult kidney tissue (23) that Kindlin-2 has consistent strong expression in renal contex. The different antibodies being used may account for the discrepancy.

Several recent studies in cell models have shown that Kindlin-2 is involved in cancer cell migration and invasion. For example, upregulated Kindlin-2 expression promoted the migration and invasion of breast cancer cells $(16,19,24)$. Kindlin-2 also promoted the invasion of gastric cancer cells mediated by tumor-associated macrophages and phosphorylation of integrin $\beta 1$ and $\beta 3(25,26)$. Inhibition of Kindlin-2 suppressed migratory/invasive properties of esophageal squamous cell carcinoma (27). In the present study, Transwell assays showed that overexpression of Kindlin-2 in 796-P cells promotes cell migration and invasion. In contrast, knockdown of Kindlin-2 in ACHN cells inhibited cell migration and invasion. Distant metastases were not found in the tumor xenograft model, but absence of tumor emboli and satellite nodules in the Kindlin-2 depleted group as compared to the control group further supports the concept that downregulation of Kindlin-2 expression suppresses invasive and metastatic capacity in RCC cells in vivo. Moreover, it was notable that knockdown of Kindlin-2 significantly inhibited ACHN cell proliferation in vitro and tumorigenesis in vivo. This is in accord with previous findings in breast cancer and pancreatic cancer $(19,28)$. However, at the same time, there are several studies showing that Kindlin-2 acts as a tumor suppressor in some cancers. It has been shown to inhibit mesenchymal cell invasion, growth and migration of colorectal cancer cells, and migration and invasion in ovarian cancer cells $(17,18,29)$. In conclusion, it appears Kindlin-2 function is cellular contextdependent.

We demonstrated that the genes involved in the Wnt signaling pathway including WNT2B, FZD3 and Axin2 are indeed downregulated by Kindlin-2 depletion through analyzing gene expression profiles of Kindlin-2 depleted RCC cells with the Gene 1.0 ST array. The constitutive activation of Wnt signaling cascades plays a critical role in the progression of RCC. Canonical Wnt ligands bind to FZD (frizzled) family receptors and the LRP5/LRP6 co-receptor, which stabilizes $\beta$-catenin. After stabilization $\beta$-catenin enters the nucleus and interacts with the members of the LEF/TCF family, resulting in a functional transcription factor complex with resultant expression of downstream target genes (Myc, cyclin D1, MMP7, Axin2, and so on) (30). There are several lines of evidence which implicate the Wnt signaling pathway in RCC. Wnt10A was shown to promote RCC carcinogenesis and progression by activating the Wnt/ $\beta$-catenin pathway (31). In addition, $\beta$-catenin is the key molecule in the pathogenesis of RCC. Elevation of the $\beta$-catenin expression level induced renal tumors in a mouse model (32). It is well known that the defects in VHL tumor suppressor gene contribute to most sporadic CCRCC. Peruzzi et al found that $V H L$ loss in CCRCC may activate oncogenic $\beta$-catenin signaling, leading to promoted tumor cell motility and invasiveness (33). Targeting the Wnt signaling pathway may have potential as a therapeutic modality for RCC. A very recent report has shown that several drugs including ethacrynic acid, ciclopirox olamine and piroctone 
olamine may induce suppression of RCC in part due to inhibition of Wnt signaling (34).

In addition, Kindlin-2 has been shown to be involved in tumor progression through regulation of the Wnt pathway. For example, the Kindlin-2- $\beta$-catenin-TCF4 tripartite complex promotes Wnt target gene expression and in turn regulates breast cancer cell invasion (16). However, another study showed that Kindlin-2 inhibited the growth and migration of colorectal cancer cells by promoting the ubiquitination of $\beta$-catenin (29) In the present study we demonstrated Kindlin-2 is involved in Wnt signaling activation in CCRCC by immunostaining of $\beta$-catenin, active $\beta$-catenin, TCF4 and Snail. In addition, IHC analysis in xenograft tumors further demonstrated that expression of $\beta$-catenin is markedly reduced by Kindlin- 2 knockdown. It is known that glycogen synthase kinase $3 \beta$ (GSK3 $\beta$ ) which is a negative regulator of Wnt signaling, initiates degradation of $\beta$-catenin. Wnt signaling can be activated using the GSK3 $\beta$ inhibitor $\mathrm{LiCl}$, whereas expression of $\beta$-catenin, active $\beta$-catenin, and TCF4 was significantly inhibited by Kindlin- 2 knockdown, even in the presence of $\mathrm{LiCl}$. These findings argue that Kindlin-2 is required for Wnt pathway activation. However, its role in activating the Wnt signaling pathway is independent of the Kindlin-2/ILK interaction. The underlying mechanism by which Kindlin-2 regulates the Wnt signaling pathway thus warrants future investigation.

In conclusion, in this study we demonstrate that high expression of Kindlin-2 is associated with high-grade tumors and lymph node metastasis of CCRCC; the presence of Kindlin-2 expression is associated with short survival in CCRCC patients, although it is not an independent prognostic factor; Kindlin-2 promotes CCRCC cell migration, invasion and proliferation by activating the Wnt signaling pathway.

\section{Acknowledgements}

This study was supported by grants from the ministry of Science and Technology of China 2016YFC1302103, 2015CB553906, 2013CB910501, and the National Natural Science Foundation of China grants 81230051, 81472734, 31170711, 81321003 and 30830048, Beijing Natural Science Foundation grant 7120002, the 111 Project of the Ministry of Education, Peking University grants BMU20120314 and BMU20130364, and a Leading Academic Discipline Project of Beijing Education Bureau to H.Z.

\section{References}

1. Siegel RL, Miller KD and Jemal A: Cancer statistics, 2015. CA Cancer J Clin 65: 5-29, 2015.

2. Ye DW, Eto M, Chung JS, Kimura G, Chang W-C, Chang Y-H, Pang S-T, Lee JL, Niu Y, Gurney H, et al: Use of targeted therapies for advanced renal cell carcinoma in the Asia-Pacific region: Opinion statement from China, Japan, Taiwan, Korea, and Australia. Clin Genitourin Cancer 12: 225-233, 2014.

3. Patel AR, Prasad SM, Shih YC and Eggener SE: The association of the human development index with global kidney cancer incidence and mortality. J Urol 187: 1978-1983, 2012.

4. Delahunt B: Advances and controversies in grading and staging of renal cell carcinoma. Mod Pathol 22 (Suppl 2): S24-S36, 2009

5. Motzer RJ,Escudier B, Oudard S, Hutson TE, Porta C, Bracarda S, Grünwald V, Thompson JA, Figlin RA, Hollaender N, et al: Phase 3 trial of everolimus for metastatic renal cell carcinoma: Final results and analysis of prognostic factors. Cancer 116: 4256-4265, 2010.
6. Sternberg CN, Davis ID, Mardiak J, Szczylik C, Lee E, Wagstaff J, Barrios CH, Salman P, Gladkov OA, Kavina A, et al: Pazopanib in locally advanced or metastatic renal cell carcinoma: Results of a randomized phase III trial. J Clin Oncol 28: 1061-1068, 2010.

7. Rini BI, Escudier B, Tomczak P, Kaprin A, Szczylik C, Hutson TE, Michaelson MD, Gorbunova VA, Gore ME, Rusakov IG, et al: Comparative effectiveness of axitinib versus sorafenib in advanced renal cell carcinoma (AXIS): A randomised phase 3 trial. Lancet 378: 1931-1939, 2011.

8. Mancini V, Battaglia M, Ditonno P, Palazzo S, Lastilla G, Montironi R, Bettocchi C, Cavalcanti E, Ranieri E and Selvaggi FP: Current insights in renal cell cancer pathology. Urol Oncol 26: 225-238, 2008.

9. Larjava H, Plow EF and Wu C: Kindlins: Essential regulators of integrin signalling and cell-matrix adhesion. EMBO Rep 9: 1203-1208, 2008.

10. Tu Y, Wu S, Shi X, Chen K and Wu C: Migfilin and Mig-2 link focal adhesions to filamin and the actin cytoskeleton and function in cell shape modulation. Cell 113: 37-47, 2003.

11. Gong X, An Z, Wang Y, Guan L, Fang W, Strömblad S, Jiang Y and Zhang H: Kindlin-2 controls sensitivity of prostate cancer cells to cisplatin-induced cell death. Cancer Lett 299: 54-62, 2010.

12. Shen Z, Ye Y, Dong L, Vainionpää S, Mustonen H, Puolakkainen $P$ and Wang S: Kindlin-2: A novel adhesion protein related to tumor invasion, lymph node metastasis, and patient outcome in gastric cancer. Am J Surg 203: 222-229, 2012.

13. Talaat S, Somji S, Toni C, Garrett SH, Zhou XD, Sens MA and Sens DA: Kindlin-2 expression in arsenite- and cadmiumtransformed bladder cancer cell lines and in archival specimens of human bladder cancer. Urology 77: 1507.e1-7, 2011.

14. Zhan J, Zhu X, Guo Y, Wang Y, Wang Y, Qiang G, Niu M, Hu J, Du J, Li Z, et al: Opposite role of Kindlin-1 and Kindlin-2 in lung cancers. PLoS One 7: e50313, 2012.

15. An Z, Dobra K, Lock JG, Stromblad S, Hjerpe A and Zhang H: Kindlin-2 is expressed in malignant mesothelioma and is required for tumor cell adhesion and migration. Int J Cancer 127: 1999-2008, 2010.

16. Yu Y, Wu J, Wang Y, Zhao T, Ma B, Liu Y, Fang W, Zhu W-G and Zhang H: Kindlin 2 forms a transcriptional complex with beta-catenin and TCF4 to enhance Wnt signalling. EMBO Rep 13: 750-758, 2012.

17. Ren C, Du J, Xi C, Yu Y, Hu A, Zhan J, Guo H, Fang W, Liu C and Zhang H: Kindlin-2 inhibits serous epithelial ovarian cancer peritoneal dissemination and predicts patient outcomes. Biochem Biophys Res Commun 446: 187-194, 2014.

18. Shi $\mathrm{X}$ and Wu C: A suppressive role of mitogen inducible gene-2 in mesenchymal cancer cell invasion. Mol Cancer Res 6: 715-724, 2008.

19. Zhao T, Guan L, Yu Y, Pei X, Zhan J, Han L, Tang Y, Li F, Fang $\mathrm{W}$ and Zhang $\mathrm{H}$ : Kindlin-2 promotes genome instability in breast cancer cells. Cancer Lett 330: 208-216, 2013.

20. Fukuda K, Bledzka K, Yang J, Perera HD, Plow EF and Qin J: Molecular basis of Kindlin-2 binding to integrin-linked kinase pseudokinase for regulating cell adhesion. J Biol Chem 289: 28363-28375, 2014.

21. Huet-Calderwood C, Brahme NN, Kumar N, Stiegler AL, Raghavan S, Boggon TJ and Calderwood DA: Differences in binding to the ILK complex determines kindlin isoform adhesion localization and integrin activation. J Cell Sci 127: 4308-4321, 2014.

22. Yan M, Zhang L, Wu Y, Gao L, Yang W, Li J, Chen Y and Jin X: Increased expression of Kindlin-2 is correlated with hematogenous metastasis and poor prognosis in patients with clear cell renal cell carcinoma. FEBS Open Bio 6: 660-665, 2016.

23. Zhan J, Yang M, Chi X, Zhang J, Pei XL, Ren CX, Guo YQ, Liu W and Zhang HQ: Kindlin-2 expression in adult tissues correlates with their embryonic origins. Sci China Life Sci 57: 690-697, 2014.

24. Yu Y, Wu J, Guan L, Qi L, Tang Y, Ma B, Zhan J, Wang Y, Fang W and Zhang H: Kindlin 2 promotes breast cancer invasion via epigenetic silencing of the microRNA200 gene family. Int J Cancer 133: 1368-1379, 2013.

25. Shen Z, Ye Y, Kauttu T, Seppänen H, Vainionpää S, Wang S, Mustonen $\mathrm{H}$ and Puolakkainen P: The novel focal adhesion gene Kindlin-2 promotes the invasion of gastric cancer cells mediated by tumor-associated macrophages. Oncol Rep 29: 791-797, 2013.

26. Shen Z, Ye Y, Kauttu T, Seppänen H, Vainionpää S, Wang S, Mustonen $\mathrm{H}$ and Puolakkainen P: Novel focal adhesion protein Kindlin-2 promotes the invasion of gastric cancer cells through phosphorylation of integrin beta1 and beta3. J Surg Oncol 108: 106-112, 2013. 
27. Zhang HF, Zhang K, Liao LD, Li L-Y, Du Z-P, Wu B-L, Wu J-Y, Xu X-E, Zeng F-M, Chen B, et al: miR-200b suppresses invasiveness and modulates the cytoskeletal and adhesive machinery in esophageal squamous cell carcinoma cells via targeting Kindlin-2. Carcinogenesis 35: 292-301, 2014.

28. Zhan J, Song J, Wang P, Chi X, Wang Y, Guo Y, Fang W and Zhang H: Kindlin-2 induced by TGF-beta signaling promotes pancreatic ductal adenocarcinoma progression through downregulation of transcriptional factor HOXB9. Cancer Lett 361: $75-85,2015$.

29. Ren Y, Jin H, Xue Z, Xu Q, Wang S, Zhao G, Huang J and Huang H: Kindlin-2 inhibited the growth and migration of colorectal cancer cells. Tumour Biol 36: 4107-4114, 2015.

30. Niehrs C: Function and biological roles of the Dickkopf family of Wnt modulators. Oncogene 25: 7469-7481, 2006.

31. Hsu RJ, Ho JY, Cha TL, Yu D-S, Wu C-L, Huang W-P, Chu P, Chen Y-H, Chen J-T and Yu C-P: WNT10A plays an oncogenic role in renal cell carcinoma by activating $\mathrm{WNT} /$ beta-catenin pathway. PLoS One 7: e47649, 2012.
32. Saadi-Kheddouci S, Berrebi D, Romagnolo B, Cluzeaud F, Peuchmaur M, Kahn A, Vandewalle A and Perret C: Early development of polycystic kidney disease in transgenic mice expressing an activated mutant of the beta-catenin gene. Oncogene 20: 5972-5981, 2001.

33. Peruzzi B, Athauda G and Bottaro DP: The von Hippel-Lindau tumor suppressor gene product represses oncogenic beta-catenin signaling in renal carcinoma cells. Proc Natl Acad Sci USA 103: 14531-14536, 2006.

34. Von Schulz-Hausmann SA, Schmeel LC, Schmeel FC and Schmidt-Wolf IG: Targeting the Wnt/beta-catenin pathway in renal cell carcinoma. Anticancer Res 34: 4101-4108, 2014. 